\title{
Contextos profissionais e práticas da avaliação psicológica: inquérito aos psicólogos portugueses
}

\author{
António M. Diniz'1 \\ Leandro S. Almeida \\ Lúcia G. Pais
}

\begin{abstract}
Resumo
A crescente preocupação com os aspectos técnicos e éticos da avaliação psicológica, em particular da utilização de técnicas de avaliação, tem-se reflectido na importância que lhes é internacionalmente atribuída. Neste enquadramento, procurámos descrever as percepções e as práticas dos psicólogos portugueses quanto à avaliação psicológica. Com base num inquérito nacional junto de psicólogos $(N=1259)$, respondido por 265 participantes com mais de três anos de actividade, descrevemo-los quanto ao percurso de formação, contexto profissional e utilização de técnicas de avaliação, e cruzámos as variáveis tempo e contextos de actividade com a utilização, e o valor e percepções quanto ao uso das técnicas. Destacamos a percepção positiva relativamente à formação recebida na área da avaliação psicológica, mesmo afirmando a necessidade de formação complementar ou o desconhecimento das orientações internacionais sobre o uso das provas (lacuna mais evidente no contexto escolar). As inovações passam, sobretudo, pelo recurso à informatização/novas tecnologias.

Palavras-chave: Testes psicológicos; Diagnóstico; Prática psicológica; Psicometria.
\end{abstract}

\section{Professional contexts and psychological assessment pactices: A survey among Portuguese psychologists}

\begin{abstract}
The growing attention given to the use of psychological assessment techniques is reflected on the importance attributed to it internationally. Our study is inscribed in this movement. We aim to characterize the use of psychological assessment techniques in Portugal, through data from a national survey of practicing psychologists $(N$ $=1259)$. Responses were collected from 265 participants with more than three years of activity, and analysed in terms of graduation course, professional context and use of psychological assessment techniques. Then, we cross variables such as time practice and professional contexts with data referring to the use, and to the value and perceptions about psychological assessment techniques utilisation. Results suggest a positive perception about the adequacy of academic preparation on psychological assessment, although feeling the necessity of further preparation and unknowing the international standards concerning tests utilization (namely school psychologists). The principal innovation to consider is the wider utilization informatics/new technologies.

Keywords: Psychological tests; Diagnostic; Psychological practice; Psicometrics.
\end{abstract}

\section{Introdução}

A avaliação psicológica é uma subdisciplina prática da psicologia que serve o objectivo genérico de apoio a processos de tomada de decisão. Ora, sabe-se já há muito tempo que a decisão, apesar de idealmente lógica e cientificamente sustentada, é susceptível a enviesamentos (Diniz, 2004; Salmon, 1990; Simões, 1993, 1999). No caso das provas psicológicas, os enviesamentos, os erros, podem decorrer, nomeadamente, da falta de adequação das suas características técnicas e psicométricas (AERA, APA \& NCME, 1999; Andriola, 1993; Bartram, 2001; Hambleton, 1994, 2001; Noronha, 1999; Shelley \&
Cohen, 1986) ou da sua deficiente utilização (Affonso, 2005; Fremer, 1996; Muñiz, Prieto, Almeida \& Bartram, 1999; Turner, DeMers, Fox \& Reed, 2001). Daqui decorre o crescente interesse que tem vindo a colocar-se sobre esta matéria, com a correlativa criação de grupos de trabalho institucionalmente enquadrados (Task Forces das American Psychological Association, European Federation of Professional Psychologists Association e International Test Commission), visando ao levantamento do estado da arte e orientando a construção e a utilização das provas psicológicas nos diferentes países.

Este movimento, centrado sobre a importância de uma competente e responsável utilização das provas psicológicas, teve a sua expressão mais visível a

\footnotetext{
${ }^{1}$ Endereço para correspondência:

ISPA - Instituto Superior de Psicologia Aplicada - Rua Jardim do Tabaco, 34 - 1149-041 - Lisboa - Portugal

E-mail: antonio.diniz@ispa.pt
} 
partir do final da década de 1980. Ele reflectiu-se num conjunto de trabalhos subordinados a esse tópico (AERA, APA \& NCME, 1999; Ball, Archer \& Imhof, 1994; Bartram, 2001; DeMers e colaboradores, 2000; Eignor, 2001; Fernández-Ballesteros, 1999; FernándezBallesteros e colaboradores, 2001; Fernández-Hermida \& Zaal, 2001; Hambleton, 1994, 2001; Muñiz e colaboradores, 1999, 2001; Turner e colaboradores, 2001; Tyler, 1986; Van de Vijver \& Hambleton, 1996) e no estabelecimento de regulamentação internacional específica quanto à utilização das provas psicológicas (International Test Commission, 2001), já adaptada para Portugal (Comissão para a Adaptação Portuguesa das Directizes Internacionais para a Utilização de Testes, 2003). Em relação ao Brasil, é de destacar também o esforço desenvolvido pelo Conselho Federal de Psicologia $(2003,2005)$ nesta matéria, sublinhando a necessidade de utilização restrita de provas psicológicas devidamente validadas.

Aceitando-se a avaliação psicológica enquanto a tarefa mais básica da prática profissional da psicologia (Almeida, Simões \& Gonçalves, 1995), mas não a menos complexa, e a sua importância para o diagnóstico e a intervenção (Brito \& Goulart, 2005; Pais, 2004), torna-se necessário o estudo sistemático do seu exercício. Havendo já um relevante esforço dos académicos portugueses na adaptação, validação e aferição de provas psicológicas, importa actualizar a informação quanto à forma como os psicólogos portugueses percepcionam e realizam a avaliação psicológica. $\mathrm{Na}$ sequência de estudos anteriores (Almeida \& Cruz, 1985, 1988; Cruz \& Almeida, 1987; Muñiz e colaboradores, 1999), com este artigo pretendemos caracterizar os psicólogos portugueses e as suas práticas profissionais, com particular incidência na utilização de provas no contexto da avaliação psicológica. Assim, num primeiro momento descrevemos os percursos de formação e de prática profissional. Num segundo, cruzámos variáveis relativas à utilização, e ao valor e percepções dos psicólogos quanto às técnicas de avaliação psicológica com os dados agrupados de acordo com o tempo de prática profissional (seis anos ou menos vs. quinze anos ou mais) e o contexto de actividade profissional dos psicólogos.

\section{Método}

\section{Participantes}

Tomaram parte neste estudo 265 psicólogos portugueses em exercício de profissão há, pelo menos, três anos $(M=39$ anos; leque etário 25-67), pertencendo a maioria ao género feminino $(78,9 \%)$. Os dois géneros diferem quanto à idade (mulheres, $M=38$; homens, $M=42$ ), sendo esta diferença estatisticamente significativa, $t(261)=-3,179, p<0,01$, traduzindo uma feminização crescente deste grupo profissional.

\section{Instrumento}

Partindo de questionários similares (Almeida \& Cruz, 1985; Muñiz e colaboradores, 1999; Poortinga e colaboradores, 1982), o questionário usado neste estudo inclui um primeiro grupo de questões descritivas dos respondentes (percurso de formação). $O$ segundo agrupamento de questões versava aspectos relativos à actividade profissional propriamente dita (contexto de exercício). Também foi perguntado se os psicólogos exerciam uma segunda actividade profissional (embora esses dados não tenham sido tratados neste estudo). O terceiro agrupamento de questões versava aspectos relativos à avaliação psicológica no contexto da actividade profissional (utilização de técnicas/ instrumentos). $\mathrm{O}$ quarto agrupamento de questões destinava-se às opiniões e necessidades dos respondentes relacionadas com a utilização de provas psicológicas (marca distintiva/identitária da prática psicológica). Para qualquer destas actividades só eram solicitadas respostas a psicólogos que as desempenhassem há mais de três anos, o que aliás levou a eliminar alguns protocolos.

\section{Procedimento}

A definição do universo amostral que serviu de ponto de partida para a realização do inquérito fez-se através de um processo intencional de amostragem, considerando três tipos de fontes, a saber: informantes privilegiados (psicólogos) ligados a entidades/ instituições públicas e privadas onde trabalhassem psicólogos em número considerável; psicólogos que, por meio das suas relações pessoais e institucionais, pudessem funcionar como elementos de uma cadeia de tipo bola-de-neve (Atkinson \& Flint, 2001); e listas telefónicas/páginas amarelas para consultórios e empresas de psicologia.

Foram expedidos por carta (com envelope de porte pago para resposta) 1.312 questionários, dos quais foram devolvidos 35 por erros de moradas e 18 por não terem sido entregues nas cadeias de tipo bola-de-neve. Um total de 289 psicólogos responderam ao inquérito, o que corresponde a uma taxa de resposta de $23 \%$. Foram anulados 24 questionários porque respondidos por psicólogos com menos de três anos de prática profissional. Os dados recolhidos junto dos 265 respondentes foram inseridos no SPSS14.0 for Windows e trabalhados, nesse programa, recorrendo a estatísticas descritivas (ocorrências, percentagens, valores mínimos 
e máximos, médias das ordenações, e médias) e inferenciais $\left[\chi^{2}\right.$ de ajustamento $\left(\chi^{2}\right.$ ai. $)$ e teste $Z$ Kolmogorov-Smirnov para uma amostra; $\chi^{2}$ de associação ( $\left.\chi_{\text {ass. }}^{2}\right)$, teste U-Mann-Whitney e teste $t$ Student para amostras independentes] de análise dos mesmos. Os valores omissos tiveram um tratamento listwise.

\section{Resultados}

Reportando-nos à caracterização do percurso de formação e da prática profissional dos psicólogos da amostra, a sua graduação (5 anos de formação em psicologia após o $12^{\circ}$ ano do ensino secundário) ocorreu entre 1972 (dois respondentes) e 2002 (10 respondentes), oscilando entre três e 34 anos de prática profissional $(29,1 \%$ com seis anos ou menos e $33,1 \%$ com 15 anos ou mais).

Em relação à área de graduação ou préespecialização, a larga maioria reporta-se à psicologia clínica (64,3\%), seguida da educacional $(22,1 \%)$, da social/organizacional $(11,2 \%)$ e da forense $(2,3 \%)$. O valor residual que esta última apresenta poderá dever-se ao facto de esta área apenas se ter autonomizado em três cursos de graduação em psicologia e em tempo mais recente.

Questionados quanto à adequação da formação recebida na área da avaliação psicológica, a maioria considera-a adequada (61,3\%), registando-se $20,7 \%$ que a considera pouco ou nada adequada e $18 \%$ que a considera muito adequada. De acrescentar que $65,6 \%$ dos sujeitos menciona ter recebido supervisão no início da sua prática profissional.

Entretanto, um quinto (20,5\%) dos respondentes fez um curso de especialização em termos de pósgraduação, $23,6 \%$ realizou mestrado e 9,5\% doutoramento (havendo ainda 16,0\% que mencionam estar a frequentar estudos pós-graduados). De notar que nesta elevada percentagem de psicólogos pós-graduados e a frequentar pós-graduações $(69,6 \%)$ se incluem os 15,2\% dos respondentes que se encontram ligados a instituições de Ensino Superior, o que nos permite afirmar a existência de uma assinalável procura de formação pósgraduada por parte dos psicólogos portugueses.

Quanto aos contextos de actividade profissional, a maioria dos respondentes trabalha em contexto escolar $(n=57 ; 22,3 \%)$ e hospitalar (44 em hospitais e cinco em centros de saúde; 19,1\%), instituições que, tradicional e internacionalmente, mais empregam psicólogos. Um menor número de psicólogos encontra-se a trabalhar noutras instituições, como empresas $(n=15 ; 5,9 \%)$ e instituições militares/policiais $(n=20 ; 7,8 \%)$. Faça-se notar que obtivemos um número de respostas ao inquérito por parte de psicólogos a trabalhar em empresas inferior ao expectável, o que leva a relativizar os resultados encontrados para este contexto de actividade. Atente-se, ainda, que no contexto militar/policial trabalham $36,8 \%$ de respondentes graduados em psicologia educacional, $31,6 \%$ em clínica e $26,3 \%$ em social/organizacional. De salientar, também, a reduzida percentagem de respondentes que centram a sua principal actividade profissional na consulta privada $(n=24$; 9,4\%), sabendo-se, contudo, que muitos psicólogos portugueses exercem a prática privada complementarmente à sua actividade profissional numa instituição.

Ainda quanto às instituições que mais empregam psicólogos, mas mais recentemente e no nosso país (a partir da década de 1980), temos as de Ensino Superior ( $n$ $=39 ; 15,2 \%)$ e as ligadas à administração da justiça $(n=$ 27 ; 10,5\%), perfazendo cerca de um quarto dos respondentes. Convém assinalar que nas primeiras, 36 respondentes são docentes e três trabalham em gabinetes de apoio psicológico aos estudantes universitários, enquanto nas segundas instituições trabalham maioritariamente respondentes graduados em psicologia clínica $(65,4 \%)$. Justifica-se esta elevada percentagem dada a notável entrada de psicólogos no Instituto de Reinserção Social e nos serviços de educação das prisões nos primórdios destes serviços, e numa altura em que não existia, ainda, formação graduada específica para a intervenção nessa área. Por contraponto, no âmbito das instituições particulares de solidariedade social para crianças e jovens $(n=8 ; 3,1 \%)$, das autarquias $(n=5$; $2,0 \%$ ), e de instituições que lidam com a deficiência mental $(n=6 ; 2,3 \%)$, a toxicodependência $(n=3 ; 1,2 \%)$, a reabilitação profissional $(n=2 ; 0,8 \%)$ e os idosos $(n=$ 1; $0,4 \%$ ), o número de respondentes é bastante reduzido.

Quanto à idade da população-alvo da intervenção dos respondentes, 43,8\% afirmaram trabalhar com adultos, $35,0 \%$ com crianças e adolescentes e $21,2 \%$ com várias combinações destas faixas etárias. De referir, ainda, que $47,3 \%$ dos respondentes desenvolvem a sua intervenção exclusivamente ao nível individual. Por último, cerca de um terço dos respondentes $(30,6 \%)$ afirmaram ter uma segunda actividade profissional como psicólogos, complementar à actividade principal assinalada (sendo que os valores aqui analisados se reportam, apenas, à actividade principal).

Na Tabela 1 listamos as provas psicológicas mais mencionadas pelos psicólogos quando lhes foi pedido para referir, por ordem decrescente, as cinco que mais utilizavam. As provas que tiveram ocorrências residuais e que foram listadas nas últimas posições (utilização relativa menos dominante) não constam na Tabela. 
Tabela 1 - Provas psicológicas mais utilizadas pelos psicólogos

\begin{tabular}{|c|c|}
\hline Designação & Ocorrências \\
\hline \multicolumn{2}{|l|}{ Provas de Inteligência/Cognição } \\
\hline WPPSI (6) + WPPSI-R* (3) & 9 \\
\hline WISC* (59) + WISC-R (20) + WISC-III* (32) & 111 \\
\hline WAIS (39) + WAIS-R (9) + WAIS-III** (3) & 51 \\
\hline Matrizes de Raven - Standard (53) + PM-47* (11) & 64 \\
\hline Desenho da Figura Humana (Goodenough) & 13 \\
\hline Bateria de Provas de Raciocínio - BPRD* (32) & 32 \\
\hline DAT* $^{*}(6)+$ PMA $*(4)+$ GATB* (8) & 18 \\
\hline Barragem de Atenção TP* & 21 \\
\hline Figura Complexa de Rey** & 42 \\
\hline Bender (16) + Benton (5) & 24 \\
\hline \multicolumn{2}{|l|}{ Provas de Personalidade } \\
\hline Rorschach & 71 \\
\hline TAT (43) + CAT (45) & 88 \\
\hline MMPI (26) + MMPI-II (3) + MMPI-R (1) + Mini-Mult (12) & 42 \\
\hline MCMI (10) + MCMI-II (7) (Multiaxial de Millon) & 17 \\
\hline CPI (California) & 10 \\
\hline NEO-PI-R* (12) & 12 \\
\hline \multicolumn{2}{|l|}{ Provas de avaliação clínica de adultos } \\
\hline Inventários de Depressão - Beck* (13) + IACLIDE* (3) + Hamilton (2) & 18 \\
\hline \multicolumn{2}{|l|}{ Provas Expressivas } \\
\hline Desenho - Família (20) + Livre (8) + HTP (3) + Árvore (2) & 33 \\
\hline \multicolumn{2}{|l|}{ Outras provas } \\
\hline Escala de Desenvolvimento de Griffiths* & 23 \\
\hline Inventários de Interesses Vocacionais - Kuder* (9) + CDI* (2) + Holland (1) & 12 \\
\hline Escalas de Autoconceito/estima - Susan Harter* (8) + Rosenberg* (2) & 10 \\
\hline
\end{tabular}

Legenda $*=$ validada; $* *=$ validação em curso; $(n)$.

Observando a Tabela 1, destacam-se, como mais frequentemente utilizadas, as escalas de inteligência de Wechsler, principalmente a WISC (listada em primeiro lugar 35,7\% das vezes e 23,2\% em segundo), seguidas das técnicas projectivas, nomeadamente o Rorschach (listado em primeiro lugar $36,6 \%$ das vezes e $19,7 \%$ em segundo). Ainda que com menor expressão, é também assinalável a utilização da WAIS (listada quer em primeiro quer em segundo lugar 28,2\% das vezes), das Matrizes de Raven (listadas em primeiro lugar $11,5 \%$ das vezes e $23,1 \%$ em segundo), da Bateria de Provas de Raciocínio Diferencial/BPRD (listada em primeiro lugar $11,5 \%$ das vezes e $37,1 \% \mathrm{em}$ segundo), da Figura Complexa de Rey (listada em primeiro lugar 4,8\% das vezes e $14,3 \%$ em segundo), do MMPI (listado em primeiro lugar $34,6 \%$ das vezes e 15,4\% em segundo) e do Desenho da Família (listada em segundo e terceiro lugar 29,4\% das vezes).

Excluindo as provas passíveis de derivação teórica em termos de análise dos resultados (Rorschach, TAT, CAT e Desenho), as provas mencionadas que se encontram validadas para a população portuguesa correspondem, apenas, a 44\% do total das ocorrências, o que parece traduzir uma elevada utilização de provas cujos resultados não são, de todo, confiáveis. Este problema de recurso a provas não validadas é especialmente evidente para os inventários de personalidade (só o NEO-PI-R está validado), de entre os quais se destaca o uso do Mini-Mult que, mesmo no original americano, apresenta problemas de validade concorrente com o MMPI.

Tendo em conta esses aspectos, importa aceder às percepções dos inquiridos no que concerne à utilização de provas psicológicas. $\mathrm{Na}$ Tabela 2 apresentamos os resultados do cruzamento dessas percepções com os dados dos subgrupos amostrais seleccionados de acordo com o contexto de actividade (só os sete mais representados na amostra). Por questões de apresentação alguns dos valores de $\chi^{2}$ aj. surgem na Tabela 2 (tal como na Tabela 3 adiante) como redundantes em face das percentagens encontradas. 
Tabela 2 - Percepções quanto à utilização de provas psicológicas $v s$. contextos profissionais

Contexto de actividade profissional

\begin{tabular}{|c|c|c|c|c|c|c|}
\hline Escolar(a) & Hospitalar(a) & Ens. Sup.(a) & Justiça(a) & Consult.(a) & Milit./Pol.(a) & \\
\hline \multicolumn{7}{|c|}{ Nível de adequação da formação em avaliação psicológica (1 - não adequada; 4 - muito adequada) } \\
\hline & & & & & & $3=71$ \\
\hline & $Z_{\mathrm{k}-\mathrm{s}}=2,42^{* * *}$ & $Z_{\mathrm{k}-\mathrm{s}}=1,95^{* *}$ & $Z_{\mathrm{k}-\mathrm{s}}=$ & $Z_{\mathrm{k}-\mathrm{s}}$ & $Z_{\mathrm{k}-\mathrm{s}}$ & \\
\hline \multicolumn{7}{|c|}{ Conhece, ou não, as directrizes internacionais para a utilização de provas } \\
\hline não & não $=$ & $\operatorname{sim}=$ & não & กล̃o $=$ & não $=57,9 \%$ & $0 \%$ \\
\hline & & $\chi^{2}=0$, & & & & \\
\hline
\end{tabular}

A defesa internacional de boas práticas no uso de provas tem tido, ou não, eco nos psicólogos portugueses $\operatorname{sim}=50,9 \% \quad$ não $=57,8 \% \quad \operatorname{sim}=51,5 \% \quad$ não $=58,3 \% \quad$ não $=66,7 \% \quad \operatorname{sim}=61,1 \% \quad \operatorname{sim}=53,3 \%$

$\chi^{2}=0,02 \quad \chi^{2}=1,09 \quad \chi^{2}=0,03 \quad \chi^{2}=0,67 \quad \chi^{2}=2,33 \quad \chi^{2}=0,89 \quad \chi^{2}=0,07$

Eleição de necessidades para mais correcta utilização de provas: formação pós-graduada (sim ou não) não $=50,9 \% \quad$ não $=57,1 \% \quad$ não $=43,6 \% \quad \operatorname{sim}=66,7 \% \quad$ não $=50,0 \% \quad$ não $=55,0 \% \quad \operatorname{sim}=53,3 \%$

$\chi^{2}=0,02 \quad \chi^{2}=1,00 \quad \chi^{2}=0,64 \quad \chi^{2}=3,00 \quad \chi^{2}=0,00 \quad \chi^{2}=0,20 \quad \chi^{2}=0,07$

Eleição de necessidades para mais correcta utilização de provas: provas validadas Portugal (sim ou não) não $=71,9 \% \quad$ não $=61,2 \% \quad$ não $=71,8 \% \quad$ não $=70,4 \% \quad$ não $=70,8 \%$ não $=90,0 \% \quad$ não $=93,3 \%$ $\chi^{2}=10,97 * * \quad \chi^{2}=2,47 \quad \chi^{2}=7,41 * * \quad \chi^{2}=4,48^{*} \quad \chi^{2}=4,17 * \quad \chi^{2}=12,80^{* * *} \quad \chi^{2}=11,27 * *$

Eleição de necessidades para mais correcta utilização de provas: divulgação sobre provas (sim ou não) não $=93,0 \%$ não $=91,8 \% \quad$ não $=84,6 \% \quad$ não $=96,3 \% \quad$ não $=87,5 \%$ $\chi^{2}=42,12^{* * *} \quad \chi^{2}=34,31 * * * \quad \chi^{2}=18,69 * * * \quad \chi^{2}=23,15^{* * *} \quad \chi^{2}=13,50 * * * \quad$ todos não $\quad$ todos não

Eleição de necessidades para mais correcta utilização de provas: supervisão pós-graduada (sim ou não) não $=75,4 \% \quad$ não $=81,6 \% \quad$ não $=92,3 \% \quad$ não $=77,8 \% \quad$ não $=95,0 \%$ $\chi^{2}=14,75^{* * *} \quad \chi^{2}=19,61 * * * \quad \chi^{2}=27,92^{* * *} \quad \chi^{2}=8,33 * * \quad$ todos não $\quad \chi^{2}=16,20 * * * \quad$ todos não

Eleição de inovações no método dos testes: informatização/recurso a novas tecnologias (sim ou não) não $=86,0 \% \quad$ não $=69,4 \% \quad$ não $=76,9 \% \quad$ não $=85,2 \% \quad$ não $=70,0 \% \quad$ não $=60,0 \%$ $\chi^{2}=29,49 * * * \quad \chi^{2}=7,37 * * \quad \chi^{2}=11,31 * * \quad \chi^{2}=13,37 * * * \quad$ todos não $\quad \chi^{2}=3,20 \quad \chi^{2}=0,60$ Eleição de inovações no método dos testes: validações para Portugal (sim ou não)

não $=94,7 \%$ não $=95,9 \% \quad$ não $=94,9 \% \quad$ não $=85,2 \% \quad$ não $=91,7 \%$ não $=90,0 \% \quad$ não $=93,3 \%$ $\chi^{2}=45,63^{* * *} \quad \chi^{2}=41,33^{* * *} \quad \chi^{2}=31,41^{* * *} \quad \chi^{2}=13,37 * * * \quad \chi^{2}=16,67 * * * \quad \chi^{2}=12,80^{* * *} \quad \chi^{2}=11,27 * *$

Eleição de inovações no método dos testes: relatividade dos resultados face aos contextos (sim ou não) não $=94,7 \%$ não $=98,0 \%$ não $=89,7 \% \quad$ não $=95,8 \%$ $\chi^{2}=45,63^{* * *} \quad \chi^{2}=45,08^{* * *} \quad \chi^{2}=24,64 * * * \quad$ todos não $\quad \chi^{2}=20,17 * * * \quad$ todos não todos não Legenda (a) $=\chi_{\text {aj.; }}^{2}$ Ens. Sup. = Ensino Superior; Consult. = Consultório; Milit./Pol. = Militar/Policial; Empres. = Empresarial; $Z_{\mathrm{k}-\mathrm{s}}=Z$-Kolmogorov-Smirnov. $* p<0,05 * * p<0,01 * * * p<0,001$

No que diz respeito à eleição das necessidades sentidas para uma mais correcta utilização de provas, e antes de passarmos aos resultados da Tabela 2, na amostra, a mais prevalente é a de formação $(n=132)$, seguindo-se a de validações para Portugal $(n=79)$, a de supervisão pósgraduada $(n=35)$ e a de maior divulgação $(n=18)$.

Acresce, ainda, que no que respeita ao tempo de actividade profissional (seis anos ou menos vs. 15 anos ou mais, $n=164$ ), e considerando as variáveis contempladas na Tabela 2, não encontrámos diferenças na amostra quanto à avaliação que psicólogos mais e menos experientes fazem do nível de adequação da formação que receberam em avaliação psicológica na sua graduação (média das ordenações - seis anos ou menos $=75,32 ; 15$ anos ou mais $=77,62 ; \quad U=2799,0, Z=0,38, p=0,704)$. As únicas diferenças encontradas indicam que os psicólogos menos

experientes são quem mais desconhece as directrizes internacionais para a utilização de provas, $\chi^{2}$ ass. $(1, N=157)$ $=8,13, p<0,01$ (seis anos ou menos, $\operatorname{sim}=21$, não $=52$; 15 anos ou mais, $\operatorname{sim}=43$, não $=41$ ), bem como aqueles que mais referem necessidade de formação, $\chi^{2}$ ass. $(1, N=$ $164)=4,61, p<0,05$ (seis anos ou menos, $\operatorname{sim}=43$, não $=$ 34; 15 anos ou mais, $\operatorname{sim}=34$, não $=53$ ).

Como se observa na Tabela 2 , nos vários contextos de actividade profissional considerados o nível de formação em avaliação psicológica é tido como adequado, principalmente nos contextos escolar e hospitalar. Entretanto, nos diversos contextos também é maioritária (mas não estatisticamente significativa) a opinião de que a defesa internacional de boas práticas tem tido eco junto dos psicólogos portugueses. Não obstante esta avaliação positiva, na generalidade dos contextos não há diferenças

Psico-USF, v. 12, n. 1, p. 1-12, jan./jun. 2007 
entre o número de psicólogos que conhece e o que desconhece as directrizes internacionais para a utilização de provas, salvo no contexto escolar em que são em maior número os que desconhecem.

Reportando-nos, agora, às necessidades sentidas para uma mais correcta utilização de provas nos diferentes contextos, em contexto escolar só no que respeita à formação não existe diferenciação entre os psicólogos que a elegem e os que o não fazem, por contraponto às demais necessidades (Tabela 2), as quais não foram predominantemente eleitas. Isso também acontece no contexto do ensino superior e no da justiça. Note-se que, quanto a este último contexto, a diferença entre os que elegem e os que não elegem a formação é marginalmente significativa, $\chi^{2}$ ai. $(1, N=27)=3,00, p=0,083$, favorável aos que a elegem. No contexto hospitalar, são as necessidades de formação e de provas validadas para Portugal que se revelam importantes. No contexto do consultório é também a formação a necessidade mais sentida pelos inquiridos, embora nenhum tenha eleito a supervisão pósgraduada como necessidade. No contexto militar/policial é, de novo, a formação a necessidade mais sentida, sendo que nenhum dos respondentes elegeu a necessidade de divulgação de provas. Por fim, no contexto empresarial, ao padrão encontrado para o militar/policial acresce que nenhum dos respondentes elege a necessidade de supervisão pós-graduada.
Quanto às inovações que os psicólogos antecipam no "método dos testes", a informatização/ recurso a novas tecnologias aparece como a mais mencionada $(n=52)$, seguindo-se as validações para Portugal $(n=16)$ e a relatividade dos resultados diante dos contextos em que decorre a avaliação (adiante: relatividade em face de contextos) $(n=14)$. Analisando a Tabela 2, os psicólogos a trabalhar nos contextos escolar, hospitalar e do ensino superior não elegem, maioritariamente, qualquer dessas inovações. No contexto da justiça, o mesmo acontece, sendo que nenhum respondente elegeu como inovação a relatividade em face de contextos. No contexto do consultório, também se assinala que nenhum elegeu como inovação a informatização/ recurso a novas tecnologias. Nos contextos militar/policial e empresarial, só no que respeita à informatização/ recurso a novas tecnologias existe um número estatisticamente equivalente de psicólogos que elege essa inovação (ante os que o não fazem).

Vejamos, agora, o que acontece relativamente à utilização e ao valor atribuído às técnicas de avaliação psicológica por parte dos respondentes. Na Tabela 3 apresentamos os resultados obtidos resultantes do cruzamento dessas variáveis com os dados dos subgrupos amostrais seleccionados de acordo com os critérios tomados na Tabela 2.

Tabela 3 - Utilização e valor das técnicas de avaliação vs. contextos profissionais

[continua]

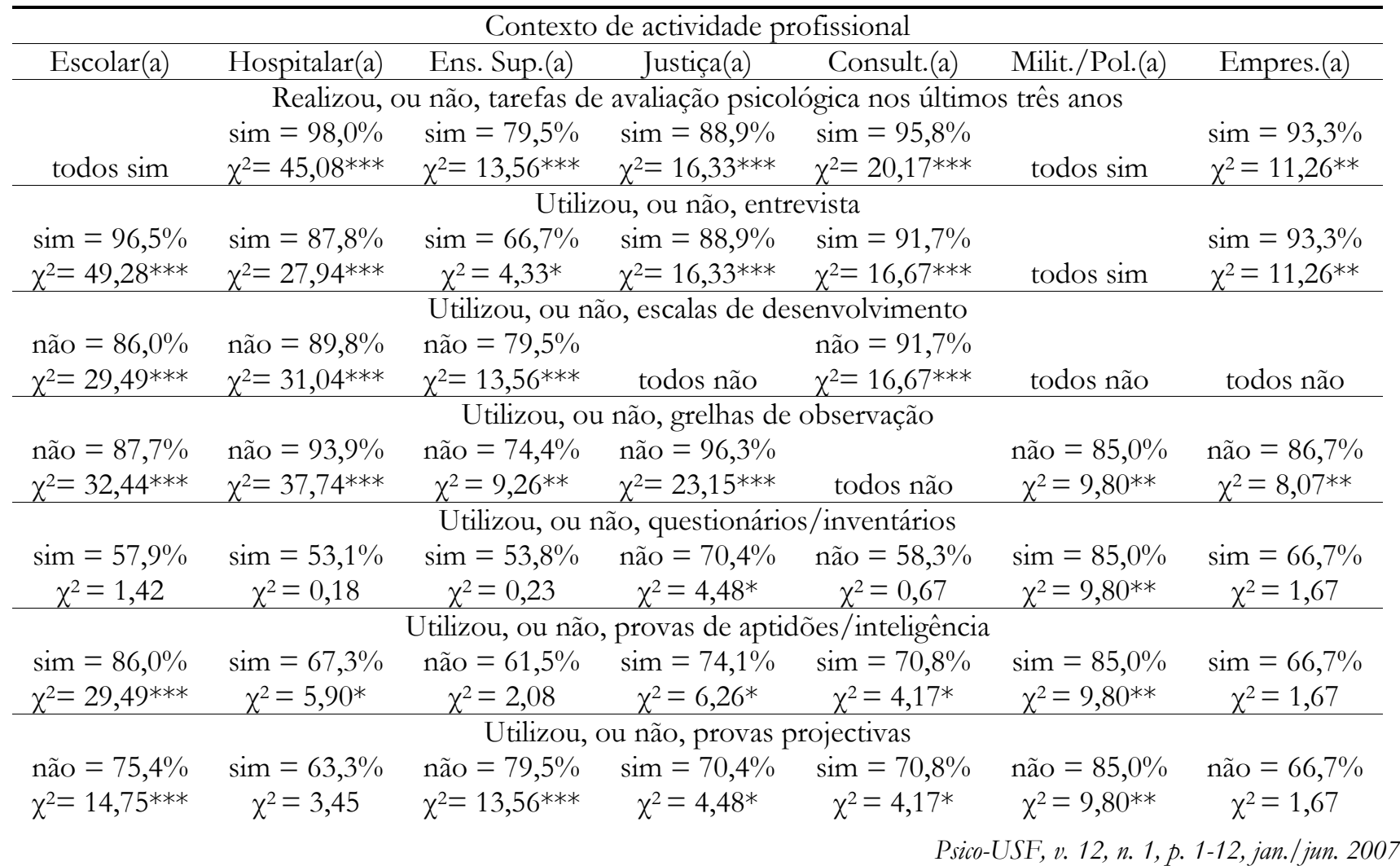




\begin{tabular}{|c|c|c|c|c|c|c|}
\hline \multicolumn{7}{|c|}{ Contexto de actividade profissional } \\
\hline Escolar(a) & Hospitalar(a) & Ens. Sup.(a) & Justiça(a) & Consult.(a) & Milit./Pol.(a) & Empres.(a) \\
\hline \multicolumn{7}{|c|}{ Frequência da utilização de provas pelos psicólogos que trabalham no contexto (1 - nunca; 4 - muito) } \\
\hline $3=64,9 \%$ & $3=62,5 \%$ & $3=59,5 \%$ & $3=55,6 \%$ & $3=41,7 \%$ & $4=55,0 \%$ & $4=57,1 \%$ \\
\hline$Z_{\mathrm{k}-\mathrm{s}}=2,93 * * *$ & $Z_{\mathrm{k}-\mathrm{s}}=2,41 * * *$ & $Z_{\mathrm{k}-\mathrm{s}}=1,91 * *$ & $Z_{\mathrm{k}-\mathrm{s}}=1,57^{*}$ & $Z_{\mathrm{k}-\mathrm{s}}=1,02$ & $Z_{\mathrm{k}-\mathrm{s}}=1,61^{*}$ & $Z_{\mathrm{k}-\mathrm{s}}=1,38^{*}$ \\
\hline \multicolumn{7}{|c|}{ Nível de preparação dos psicólogos portugueses para utilização de provas $(1$ - muito má; 5 - muito boa) } \\
\hline $3=50,9 \%$ & $3=60,4 \%$ & $3=42,1 \%$ & $3=50,0 \%$ & $3=50,0 \%$ & $3=45,0 \%$ & $4=46,7 \%$ \\
\hline$Z_{\mathrm{k}-\mathrm{s}}=1,91 * *$ & $Z_{\mathrm{k}-\mathrm{s}}=2,14 * * *$ & $Z_{\mathrm{k}-\mathrm{s}}=1,39 *$ & $Z_{\mathrm{k}-\mathrm{s}}=1,40^{*}$ & $Z_{\mathrm{k}-\mathrm{s}}=1,33$ & $Z_{\mathrm{k}-\mathrm{s}}=1,10$ & $Z_{\mathrm{k}-\mathrm{s}}=1,11$ \\
\hline \multicolumn{7}{|c|}{ A utilização de provas torna, ou não, mais precisa/rigorosa a prática psicológica } \\
\hline $\operatorname{sim}=86,0 \%$ & $\operatorname{sim}=98,0 \%$ & $\operatorname{sim}=88,9 \%$ & & $\operatorname{sim}=87,0 \%$ & $\operatorname{sim}=95,0 \%$ & \\
\hline$\chi^{2}=29,49 * * *$ & $\chi^{2}=45,08 * * *$ & $\chi^{2}=21,79 * * *$ & todos sim & $\chi^{2}=12,57 * * *$ & $\chi^{2}=16,20 * * *$ & todos sim \\
\hline \multicolumn{7}{|c|}{ A utilização de provas é, ou não, uma marca distintiva/identitária da prática psicológica } \\
\hline $\operatorname{sim}=80,7 \%$ & $\operatorname{sim}=91,5 \%$ & $\operatorname{sim}=83,8 \%$ & $\operatorname{sim}=85,2 \%$ & $\operatorname{sim}=71,4 \%$ & $\operatorname{sim}=94,7 \%$ & \\
\hline$\chi^{2}=21,49 * * *$ & $\chi^{2}=32,36^{* * *}$ & $\chi^{2}=16,89 * * *$ & $\chi^{2}=13,37 * * *$ & $\chi^{2}=3,86$ & $\chi^{2}=15,21 * * *$ & todos sim \\
\hline Legenda (a) $=$ & $c_{\text {aj. }}^{2}$ Ens. Su & $=$ Ensino Su & or; Consu & Consultóri & Milit./Pol. & Tilitar/Polic \\
\hline
\end{tabular}

No que respeita à amostra, e antes de passarmos à leitura da Tabela 3, a grande maioria dos inquiridos realizou tarefas de avaliação psicológica nos últimos três anos $(n=248)$. Quase todos usam entrevista $(n=232)$, muitos usam provas de aptidões/inteligência $(n=175)$, sendo que são muito pouco utilizadas as escalas de desenvolvimento $(n=$ 31) e as grelhas de observação $(n=32)$. Quanto à utilização de provas projectivas, a diferença entre os que o fazem $(n=110)$ e os que não o fazem $(n=155)$ esbate-se. Acresce a existência de um número de utilizadores de questionários/inventários ( $\mathrm{n}=144$ ) semelhante ao de não utilizadores $(n=121)$.

Acresce, ainda, que no que concerne ao tempo de actividade profissional, e considerando as variáveis contempladas na Tabela 3 , não encontrámos na amostra diferenças quanto à avaliação que psicólogos mais e menos experientes fazem da frequência de utilização de provas pelos psicólogos que trabalham no seu contexto de prática (média das ordenações - seis anos ou menos $=77,68 ; 15$ anos ou mais $=75,38, \mathrm{U}=2799,0, \mathrm{z}=0,37, p=0,714)$, bem como do nível de preparação dos psicólogos portugueses para a utilização de provas (média das ordenações - seis anos ou menos $=75,65 ; 15$ anos ou mais $=77,31 ; \quad U=2823,0, z=0,26, p=0,797)$. Registe-se que os psicólogos menos experientes são os que os que mais utilizam questionários/ inventários, $\chi^{2}$ ass. $(1, N=164)=4,97, p<0,05$ (seis anos ou menos, $\operatorname{sim}=53$, não $=24 ; 15$ anos ou mais, $\operatorname{sim}=45$, não $=42$ ) e os que menos utilizam escalas de desenvolvimento: esta última diferença é marginalmente significativa, $\chi^{2}{ }_{\text {ass. }}(1, N=164)=4,18$, $p=0,070$ (seis anos ou menos, $\operatorname{sim}=4$, não $=73 ; 15$ anos ou mais, $\operatorname{sim}=13$, não $=74$ ).
Verificamos, na Tabela 3, que a realização de tarefas de avaliação psicológica atravessa os diversos contextos de actividade profissional. Quanto aos instrumentos de avaliação utilizados nesses contextos, podemos afirmar que no escolar são muito utilizadas a entrevista e as provas de aptidões/inteligência, ao contrário das escalas de desenvolvimento, das grelhas de observação e das provas projectivas. Quanto aos questionários/inventários existe um número estatisticamente equivalente de psicólogos que os utiliza (face aos que o não fazem). No contexto hospitalar, utilizam muito a entrevista e menos as provas de aptidões/inteligência, por contraponto ao que acontece com as escalas de desenvolvimento e grelhas de observação. Quanto às provas projectivas, existe uma diferença marginalmente significativa, $\chi^{2}$ aj. $(1, N=49)=$ $3,45, p=0,063$, que remete para o seu uso. Quanto aos questionários/inventários existe um número estatisticamente equivalente de psicólogos que os utiliza (em relação aos que o não fazem). No contexto do ensino superior realça-se o recurso à entrevista, tal não acontecendo com as grelhas de observação e, ainda menos, com as escalas de desenvolvimento e as provas projectivas. Quanto aos questionários/inventários e às provas de aptidões/inteligência não existe diferenciação entre os psicólogos que os utilizam e os que o não fazem. No contexto da justiça, os psicólogos recorrem também muito à entrevista, mas menos às provas de aptidões/inteligência e às provas projectivas. Ninguém utiliza escalas de desenvolvimento, poucos utilizam questionários/inventários e ainda menos grelhas de observação. Em consultório, o padrão de utilização da entrevista, das provas de aptidões/inteligência e das provas projectivas é igual ao do contexto antes referido. Ninguém utiliza grelhas de observação (único contexto 
em que tal acontece) e muito poucos escalas de desenvolvimento. Quanto aos questionários/inventários não existe diferenciação entre os psicólogos que os utilizam e os que o não fazem. No contexto militar/policial, todos os psicólogos afirmam utilizar a entrevista (único contexto em que tal acontece) e nem um escalas de desenvolvimento. É frequente o recurso a questionários/inventários, bem como a provas de aptidões/inteligência, por contraponto ao que acontece com as grelhas de observação e com as provas projectivas. Por último, nas empresas, mantém-se a tónica na utilização da entrevista, não se diferenciando os psicólogos pela utilização, ou não, de questionários / inventários, provas de aptidões/inteligência e provas projectivas. Ninguém utiliza escalas de desenvolvimento e poucos recorrem a grelhas de observação.

Podemos referir, ainda, que a opinião dos psicólogos é a de que a frequência de utilização de provas é predominantemente elevada no seu contexto de prática profissional (em particular no escolar e no hospitalar), salvo para aqueles que trabalham em consultório. No que respeita à preparação dos psicólogos portugueses para utilizar provas, aqueles que trabalham em consultório (diferença marginalmente significativa para esta variável, z-Kolmogorov-Smirnov $=1,33, p=0,059)$ e nos contextos militar $/$ policial $\mathrm{e}$ empresarial (leque de respostas variou apenas entre $2 \mathrm{e}$ 4) são a excepção relativamente aos dos demais contextos, os quais consideram como predominantemente boa a preparação dos psicólogos portugueses para a sua utilização. Por outro lado, a grande maioria dos psicólogos nos diversos contextos é de opinião que a utilização de provas torna mais precisa/rigorosa a prática psicológica, considerando-a também uma marca distintiva/identitária da mesma. Porém, para os psicólogos que trabalham em consultório a utilização de provas não constitui necessariamente uma marca distintiva/identitária da prática psicológica, uma vez que encontrámos uma diferença marginalmente significativa nesta variável, $\chi_{\text {aj. }}^{2}(1, N=21)=3,86, p=0,050$.

\section{Discussão}

Diante do primeiro objectivo deste estudo caracterizar a prática dos psicólogos portugueses -, importa referir a juventude dos profissionais de psicologia em Portugal, havendo menos que um terço desses profissionais com mais de 15 anos de prática. $\mathrm{O}$ aparecimento tardio dos cursos de psicologia nas universidades portuguesas (final da década de 1970) e o aumento exponencial dos cursos de psicologia oferecidos em instituições privadas do ensino superior em Portugal, justificarão essas taxas. Entretanto, o assinalável aumento de procura de formação pósgraduada pelos psicólogos é concomitante ao aumento da oferta de formação a esse nível e à percepção pelos profissionais da necessidade de formação e actualização contínua. Por outro lado, como na generalidade dos países, a larga maioria dos psicólogos trabalha em contextos de clínica/saúde e de educação, acompanhando o volume diferenciado de solicitações sociais pelas diferentes áreas de especialização dos psicólogos e, ainda, o desenvolvimento apenas recente de formações especializadas noutras áreas, por exemplo a psicologia forense. Aliás, importa referir que a generalidade do exercício profissional dos psicólogos portugueses ocorre em instituições públicas, em particular na saúde e na educação, acrescida geralmente de forma complementar - da prática privada (gabinetes, consultórios, centros). Por último, assiste-se a uma ainda reduzida percentagem de psicólogos exercendo a sua actividade profissional junto da população idosa, o que parece não acompanhar a preocupação crescente de intervenção social junto deste grupo e também os dados demográficos referentes ao aumento da taxa de envelhecimento da população nos países europeus.

Descrevendo as percepções e as práticas dos psicólogos portugueses no que diz respeito à avaliação psicológica, a larga maioria considera a formação recebida como adequada (apenas um quinto dos respondentes aponta fragilidades nessa formação). Estes valores acompanham a percepção por parte das instituições de formação da mais-valia desta área na formação académica inicial dos psicólogos (Simões, 1999). Entretanto, podendo parecer óbvia e expectável a maior necessidade de formação pós-graduada apontada pelos psicólogos mais inexperientes, já parece menos adequado que os jovens psicólogos mais refiram desconhecer as directrizes internacionais que orientam as práticas da avaliação psicológica. E isso relaciona-se, também, com o problema inerente às práticas da avaliação psicológica que decorre do número ainda elevado de provas utilizadas não previamente validadas na população portuguesa. Esta situação apresenta maior incidência e gravidade no que diz respeito aos questionários de personalidade onde, após uma tradução mais ou menos cuidada por parte dos serviços de psicologia nas diferentes instituições, se avança numa aplicação pouco criteriosa e precisa de tais instrumentos. A situação é já menos frequente nos testes de inteligência e de aptidões intelectuais, muito embora só muito recentemente se avançou para a aferição nacional da escala de Wechsler para a população adulta (aliás, ao nível da infância e da adolescência, os psicólogos portugueses têm à sua 
disposição um maior número de provas validadas, incluindo a WPPSI-R e a WISC-III).

Tudo isso torna clara a necessidade de sensibilizar os psicólogos portugueses quanto às boas práticas na utilização de provas e de desenvolver mais estudos de validação de provas em Portugal, especialmente de personalidade, acompanhadas de mais informação/divulgação sobre provas já adaptadas/ validadas em Portugal junto dos psicólogos portugueses e de um incremento da formação sobre a avaliação psicológica e a utilização apropriada de provas psicológicas nesse contexto. Por razões análogas a estas, o Conselho Federal de Psicologia brasileiro (2003, 2005) assumiu um papel relevante nestes domínios, estimulando a validação de provas psicológicas como ponto prévio à sua utilização e invertendo o que vinha acontecendo nesse país desde há décadas (Andriola, 1993; Noronha, 1999; Sisto, Codennotti, Costa \& Nascimento, 1979). Ainda quanto à formação em avaliação psicológica, ela é tida como necessidade principal pela generalidade dos psicólogos, com excepção dos que trabalham em contexto escolar e, por razões compreensíveis, no do ensino superior. Em relação às inovações antecipadas para o "método dos testes", aquela que se revela mais importante é a do recurso à informática/novas tecnologias, tomada enquanto elemento de suporte do mesmo. Todavia, ela só tem alguma expressão nos contextos militar/policial e empresarial, sendo que os resultados relativos a este último devem ser prudentemente considerados, como já antes foi referido. Observe-se que essa inovação não tem qualquer expressão no contexto do trabalho em consultório ou clínica privada, o que poderá relacionarse com a possibilidade de muitos dos psicólogos desenvolverem sobretudo tarefas de acompanhamento/ psicoterapia. Por último, os psicólogos dos contextos escolar, hospitalar e ensino superior não elegeram, maioritariamente, qualquer das inovações elicitadas pelos psicólogos na sua globalidade. Isso poderá denunciar alguma falta de motivação em face destas matérias, mesmo no contexto do ensino superior.

No tocante às tarefas de avaliação psicológica, elas atravessam os diversos contextos de actividade profissional, em particular a entrevista, os questionários/inventários e as provas de aptidões/ inteligência, por oposição à utilização de grelhas de observação e às escalas de desenvolvimento. Ainda a este propósito, os psicólogos menos experientes são os que mais utilizam questionários e os que menos utilizam escalas de desenvolvimento. Este resultado pode estar associado a menos dados normativos para apoio à interpretação dos resultados obtidos com esses últimos instrumentos, pois acabam por exigir maior experiência

Psico-USF, v. 12, n. 1, p. 1-12, jan./jun. 2007 profissional por parte do psicólogo. Como era de esperar, as provas projectivas são mais usadas em contextos como o hospitalar, o da justiça e no consultório. Entretanto, o uso dessas provas, nomeadamente o Rorschach, suplanta claramente a utilização dos inventários de personalidade. A saber, isso pode relacionar-se com aspectos ligados ao percurso de graduação dos psicólogos portugueses, conforme o encontrado por Noronha e colaboradores (2002) em estudantes de psicologia brasileiros. Verificase, ainda, que, salvo no contexto de trabalho em consultório, a opinião dos psicólogos é a de que as provas psicológicas são bastante utilizadas nos seus contextos de prática profissional, relacionando-se com o facto de acharem que isso torna mais preciso/rigoroso o trabalho em psicologia, incluindo, aqui, a consulta privada. Pensam, ainda, que a necessária preparação para o fazer está assegurada. Essa afirmação deve ser relativizada para os que trabalham em consultório, em contexto militar/policial e no empresarial. Por último, o único contexto em que a utilização de provas não é considerada como sendo necessariamente uma marca distintiva/identitária da prática psicológica é o do consultório, o que podemos relacionar com o facto de esses psicólogos pertencerem ao único contexto em que a frequência de utilização de provas psicológicas não foi estatisticamente significativa. Acresce que a valorização que os psicólogos portugueses fazem do rigor/precisão que os testes trazem para a prática psicológica, bem como a opinião generalizada de que a sua utilização é uma marca distintiva/identitária desse trabalho, vai no mesmo sentido do encontrado no Brasil por Oliveira, Noronha e Dantas (2006).

Descritos e comentados esses resultados, importa não terminar sem alertar para algumas questões metodológicas do presente estudo. Em primeiro lugar, tendo-se registado uma percentagem de respondentes ao inquérito em torno dos $20 \%$ - valor que tem sido também encontrado noutros estudos similares - é certo que sempre podemos questionar a representatividade da amostra recolhida. Os valores percentuais obtidos na amostra referentes a algumas variáveis de caracterização social e profissional (género de pertença, idade, actividade...), salvo o caso dos psicólogos nas empresas, não se afastam, no entanto, daqueles que se poderiam inferir, ainda que impressivamente, como descrevendo os psicólogos em Portugal. Um segundo aspecto reporta-se à existência de um menor número de respondentes em certas áreas da prática profissional, mesmo correspondendo à realidade, o que não deixa de dificultar algumas análises estatísticas comparando subgrupos de psicólogos segundo tais variáveis, 
nomeadamente no que respeita ao $\chi^{2}$. Ainda assim, parece-nos que este trabalho oferece uma panorâmica actualizada das percepções e das práticas dos psicólogos portugueses em relação à avaliação psicológica.

\section{Referências}

AERA, APA \& NCME - American Educational Research Association, American Psychological Association \& National Council on Measurement in Education (1999). Standars for educational and psychological testing. New York: American Educational Research Association.

Affonso, R. M. L. (2005). A importância da epistemologia no ensino da avaliação psicológica no processo psicodiagnóstico. Avaliação Psicológica, 4(2), 183-193.

Almeida, L. S. \& Cruz, O. M. R. (1985). Utilização dos testes psicológicos: resultados de um inquérito junto dos psicólogos portugueses. Jornal de Psicologia, 4(5), 11-18.

Almeida, L. S. \& Cruz, O. M. R. (1988). O uso dos testes como revelador dos modelos de prática psicológica em Portugal. Psicologia: Teoria e Pesquisa, 4(3), 207-224.

Almeida, L. S., Simões, M. R \& Gonçalves, M. M. (1995). Provas psicológicas em Portugal: Situação actual, limitações e desafios. Em L. S. Almeida, M. M. Gonçalves \& M. R. Simões (Eds.). Provas psicológicas em Portugal (pp. I-IX). Braga: APPORT.

Andriola, W. A. (1993). Os testes psicológicos no Brasil: problemas, pesquisas e perspectivas para o futuro. Em L. S. Almeida \& I. S. Ribeiro (Orgs.). Avaliação Psicológica: Formas e Contextos (v. 3, pp. 7782). Braga: APPORT.

Atkinson, R. \& Flint, J. (2001). Accessing hidden and hard-to-reach populations: Snowball research strategies. Social Research Update [On-line], 33. Disponível em: <http://www.soc.surrey.ac.uk/sru/ SRU33.html>. Acesso em: nov. 2005.

Ball, J. D., Archer, R. P. \& Imhof, E. A. (1994). Time requirements of psychological testing: A survey of practitioners. Journal of Personality Assessment, 63(2), 239-249.

Bartram, D. (2001). Guidelines for test users: A review of national and international initiatives. European Journal of Psychological Assessment, 17(3), 173-186.

Brito, D. P. \& Goulart, I. B. (2005). Avaliação psicológica e prognóstico de comportamento desviante numa corporação militar. Psico-USF, 10(2), 149-160.

Comissão para a Adaptação Portuguesa das Directrizes Internacionais para a Utilização de Testes (2003). Directrizes internacionais para a utilização de testes [Online]. Disponível em: <http://www.intestcom.org/ itc_projects.htm>. Acesso em: ago. 2003.

Conselho Federal de Psicologia (2003). Resolução n ${ }^{\circ}$ 2/2003 [On-line]. Disponível em: <http:// www.pol.org.br>. Acesso em: ago. 2006.

Conselho Federal de Psicologia (2005). Relação dos testes psicológicos avaliados publicada pelo CFP [On-line]. Disponível em: <http://www.pol.org.br> . Acesso em: ago. 2006.

Cruz, O. M. R. \& Almeida, L. S. (1987). A utilização dos testes psicológicos em Portugal: análise segundo a formação e o domínio de prática profissional. Jornal de Psicologia, 6(1), 13-19.

DeMers, S. Y., Turner, S. M. (Cochairs), Anberg, M., Foote, W., Hough, L., Ivnik, R., Meier, S., Moreland, K. \& Rey-Casserly, C. M. (2000). Report of the task force on test user qualifications. Washington, DC: Practice and Science Directorates, American Psychological Association.

Diniz, A. M. (2004). Sobre essas coisas a que chamamos crenças. Lisboa: Climepsi.

Eignor, D. (2001). Standards for the development and use of tests: The standards for educational and psychological testing. European Journal of Psychological Assessment, 17(3), 157-173.

Fernández-Ballesteros， R. (1999). Psychological assessment: Futures, challenges and progresses. European Psychologist, 4, 248-262.

Fernández-Ballesteros, R., De Bruyn, E. E. J., Godoy, A., Hornke, L. F., Ter Laak, J., Vizcarro, C., Westhoff, K., Westmeyer, H. \& Zaccagnini, J. L. (2001). Guidelines for the assessment process (GAP): A proposal for discussion. European Journal of Psychological Assessment, 17(3), 187-200.

Fernández-Hermida, J. R. \& Zaal, J. N. (2001). Testing practices in European countries. European Journal of Psychological Assessment, 17(3), 201-211.

Fremer, J. (1996). Promoting high standards for test use: Developments in the United States. European Journal of Psychological Assessment, 12(2), 160-168.

Hambleton, R. K. (1994). Guidelines for adaptating educational and psychological tests: A progress report. European Journal Psychological Assessment, 10(3), 229-244. 
Hambleton, R. K. (2001). The next generation of the ITC test translation and adaptation guidelines. European Journal Psychological Assessment, 17(3), 164172.

International Test Commission (2001). ITC guidelines on test use [On-line]. Disponível em: <http://www.intestcom.org/ itc_projects.htm>. Acesso em: ago. 2003.

Muñiz, J., Prieto, G., Almeida, L. S. \& Bartram, D. (1999). Tests use in Spain, Portugal and Latin American Countries. European Journal of Psychological Assessment, 15(2), 151-157.

Muñiz, J., Bartram, D., Evers, A., Boben, A., Matesic, K., Glabeke, K., Ferández-Hermida, J. R. \& Zaal, J. N. (2001). Testing practices in European Countries. European Journal of Psychological Assessment, 17(3), 201211.

Noronha, A. P. P. (1999). Avaliação psicológica segundo psicólogos: usos e problemas com ênfase nos testes. Tese de doutoramento. Campinas: Pontifícia Universidade Católica de Campinas.

Noronha, A. P. P., Sartori, F. A., Freitas, F. A. \& Ottati, F. (2001). Informações contidas nos manuais de testes de inteligência publicados no Brasil. Psicologia em Estudo, 6, 101-106.

Noronha, A. P. P., Oliveira, A. F., Cobêro, C., Paula, L. M., Cantalice, L. M., Guerra, P. B. C., Martins, R. M. M. \& Felizatti, R. (2002). Instrumentos psicológicos mais conhecidos por estudantes do sul de Minas Gerais. Avaliação Psicológica, 2, 151-158.

Oliveira, K. L., Noronha, A. P. P. \& Dantas, M. A. (2006). Instrumentos psicológicos: estudo comparativo entre estudantes e profissionais cognitivo-comportamentais. Estudos de Psicologia, 23(4), 359-367.

Pais, L. G. (2004). Uma história das ligações entre a psicologia e o direito em Portugal: perícias psiquiatricas médico-legais e perícias sobre a personalidade como analisadores. Dissertação de doutoramento. Porto: Universidade do Porto.
Poortinga, Y. H., Coetsier, P., Meuris, G., Miller, K. M., Samsonowitz, V., Seisdedos, N. \& Schlegel, J. (1982). A survey of attitudes towards tests among psychologists in six western European Countries. International Review of Applied Psychology, 31, 7-34.

Salmon, W. C. (1990). Four decades of scientific explanation. Minneapolis, MN: University of Minnesota Press.

Shelley, D. \& Cohen, D. (1986). Testing psychological tests. London: Cromm Helm.

Simões, M. R. (1993). Heurísticas, enviesamentos e erros inferenciais na mecânica da avaliação psicológica. Análise Psicológica, 11(2), 253-266.

Simões, M. R. (1999). O ensino e a aprendizagem da avaliação psicológica: o caso da avaliação da personalidade. Psychologica, 22, 135-172.

Sisto, F. F., Codennotti, N., Costa, C. A. J. \& Nascimento, T. C. N. (1979). Testes psicológicos no Brasil: que medem realmente. Educação e Sociedade, 2, 152-165.

Turner, S. M., DeMers, S. T., Fox, H. R. \& Reed, G. M. (2001). APA's guidelines for test user qualifications. American Psychologist, 56, 1099-1113.

Tyler, B. (1986). The use of tests by psychologists: Report on a survey of British Psychological Society members. Bulletin of the International Test Commission, 22, 7-18.

Van de Vijver, F. \& Hambleton, R. K. (1996). Translating tests: Some practical guidelines. European Psychologist, 1(2), 89-99.

Sobre os autores:

António M. Diniz é doutor em Psicologia da Educação pela Universidade do Minho, professor auxiliar no ISPA. Investiga nas áreas de avaliação psicológica, construção e adaptação de provas psicológicas, métodos quantitativos e qualitativos aplicados ao estudo do jovem adulto universitário e do adulto idoso. 
Leandro S. Almeida é doutor em Psicologia da Educação pela Universidade do Porto e professor catedrático do Instituto de Educação e Psicologia da Universidade do Minho. Investiga nas áreas da cognição, aprendizagem, avaliação psicológica, construção e validação de instrumentos. É autor de provas de avaliação cognitiva.

Lúcia G. Pais é doutora em Psicologia pela Universidade do Porto e professora auxiliar no ISCPSI - Instituto Superior de Ciências Policiais e Segurança Interior e ISPA. Investiga nas áreas de avaliação psicológica e decisão clínica em contexto forense, decisão judicial, história das ligações entre Psicologia e Direito, estudos de personagens e fontes documentais ligadas ao fenómeno criminal. 